\title{
ON THE COMPLEXITY OF THE 3-KERNEL PROBLEM IN SOME CLASSES OF DIGRAPHS ${ }^{1}$
}

\author{
Pavol Hell and César Hernández-Cruz \\ School of Computing Science \\ Simon Fraser University \\ Burnaby, B.C., Canada V5A $1 S 6$ \\ e-mail: pavol@sfu.ca \\ chernand@sfu.ca
}

\begin{abstract}
Let $D$ be a digraph with the vertex set $V(D)$ and the arc set $A(D)$. A subset $N$ of $V(D)$ is $k$-independent if for every pair of vertices $u, v \in N$, we have $d(u, v), d(v, u) \geq k$; it is l-absorbent if for every $u \in V(D)-N$ there exists $v \in N$ such that $d(u, v) \leq l$. A $k$-kernel of $D$ is a $k$-independent and $(k-1)$-absorbent subset of $V(D)$. A 2-kernel is called a kernel.

It is known that the problem of determining whether a digraph has a kernel ("the kernel problem") is NP-complete, even in quite restricted families of digraphs. In this paper we analyze the computational complexity of the corresponding 3-kernel problem, restricted to three natural families of digraphs.

As a consequence of one of our main results we prove that the kernel problem remains NP-complete when restricted to 3-colorable digraphs.

Keywords: kernel, 3-kernel, NP-completeness, multipartite tournament, cyclically 3 -partite digraphs, $k$-quasi-transitive digraph.
\end{abstract}

2010 Mathematics Subject Classification: 05C20.

\section{REFERENCES}

[1] J. Bang-Jensen and G. Gutin, Digraphs (Springer-Verlag, Berlin Heidelberg New York, 2002).

[2] J. Bang-Jensen and J. Huang, Quasi-transitive digraphs, J. Graph Theory 20 (1995) 141-161. doi:10.1002/jgt.3190200205

\footnotetext{
${ }^{1}$ This research was partially supported by the NSERC Discovery Grant of the first author, and the CONACYT postdoctoral fellowship of the second author.
} 
[3] C. Berge, Graphs (North-Holland, Amsterdam, 1985).

[4] C. Berge and P. Duchet, Recent problems and results about kernels in directed graphs, Discrete Math. 86 (1990) 27-31. doi:10.1016/0012-365X(90)90346-J

[5] J.A. Bondy and U.S.R. Murty, Graph Theory (Springer-Verlag, Berlin Heidelberg New York, 2008).

[6] V. Chvátal, On the computational complexity of finding a kernel, Technical Report Centre de Recherches Mathématiques, Université de Montréal CRM-300 (1973).

[7] A.S. Fraenkel, Planar kernel and Grundy with $d \leq 3, d^{+} \leq 2, d^{-} \leq 2$ are $N_{-}$ complete, Discrete Appl. Math. 3 (1981) 257-262. doi:10.1016/0166-218X(81)90003-2

[8] H. Galeana-Sánchez, I.A. Goldfeder and I. Urrutia, On the structure of 3-quasitransitive digraphs, Discrete Math. 310 (2010) 2495-2498. doi:10.1016/j.disc.2010.06.008

[9] H. Galeana-Sánchez and C. Hernández-Cruz, $k$-kernels in multipartite tournaments, AKCE Int. J. Graphs Comb. 8 (2011) 181-198.

[10] H. Galeana-Sánchez and C. Hernández-Cruz, On the existence of k-kernels digraphs and in weighted digraphs, AKCE Int. J. Graphs Comb. 7 (2010) 201-215.

[11] H. Galeana-Sánchez and C. Hernández-Cruz, Cyclically k-partite digraphs and $k$ kernels, Discuss. Math. Graph Theory 31 (2011) 63-78. doi:10.7151/dmgt.1530

[12] H. Galeana-Sánchez and C. Hernández-Cruz, $k$-kernels in generalizations of transitive digraphs, Discuss. Math. Graph Theory 31 (2011) 293-312. doi:10.7151/dmgt.1546

[13] H. Galeana-Sánchez, C. Hernández-Cruz and M.A. Juárez-Camacho, On the existence and number of $(k+1)$-kings in $k$-quasi-transitive digraphs, Discrete Math. 313 (2013) 2582-2591.

[14] C. Hernández-Cruz and H. Galeana-Sánchez, $k$-kernels in $k$-transitive and k-quasitransitive digraphs, Discrete Math. 312 (2012) 2522-2530. doi:10.1016/j.disc.2012.05.005

[15] M. Kwaśnik, A. Włoch and I. Włoch, Some remarks about $(k, l)$-kernels in directed and undirected graphs, Discuss. Math. 13 (1993) 29-37.

[16] M. Richardson, On weakly ordered systems, Bull. Amer. Math. Soc. 52(2) (1946) 113-116. doi:10.1090/S0002-9904-1946-08518-3

[17] A. Sánchez-Flores, A counterexample to a generalization of Richardson's theorem, Discrete Math. 65 (1987) 319-320. doi:10.1016/0012-365X(87)90064-1 